International Journal of Pure and Applied Mathematics

Volume 105 No. 1 2015, 7-18

ISSN: 1311-8080 (printed version); ISSN: 1314-3395 (on-line version)

url: http://www.ijpam.eu

doi: http://dx.doi.org/10.12732/ijpam.v105i1.2

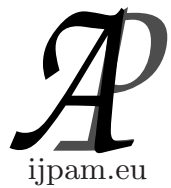

\title{
bI-OPEN SETS IN IDEAL BITOPOLOGICAL SPACES
}

\author{
Diganta Jyoti Sarma \\ Central Institute of Technology, Kokrajhar \\ BTAD, ASSAM-783370, INDIA
}

\begin{abstract}
The aim of this article is to introduce and study the concept of $b I$-open sets with respect to an ideal in bitopological spaces and to investigate some properties. Moreover, the concept of $b I$-continuous functions have also been introduced.
\end{abstract}

AMS Subject Classification: 54A10, 54C08, 54C10, 54D15

Key Words: bitopological spaces, $(i, j)$-b-open sets, $(i, j)$-bI-closed sets, $(i, j)$ $b I$-open sets

\section{Introduction}

The concept of bitopological spaces $\left(X, \tau_{1}, \tau_{2}\right)$, equipped with topologies $\tau_{1}$ and $\tau_{2}$ was introduced by Kelly [10]. The concept of ideals has been applied and studied by Kuratowski [11], Vaidyanath-asamy [15], Jankovic and Hamlett [9] and many others. An ideal $I$ on a non-empty set $X$ is a collection of subsets of $X$ satisfying (i) $A \in I$ and $B \subset A$ implies $B \in I$ and (ii) $A \in I$ and $B \in I$ implies $A \cup B \in I$. If $I$ is an ideal on $X$, then $\left(X, \tau_{1}, \tau_{2}, I\right)$ is said to be an ideal bitopological space. Andrijevic [5] introduced the notion of $b$-open sets in topological spaces. Further, Al-Hawary and Al-Omari [4] extended this notion to bitopological spaces. In 2007, Caksu Guler and Aslim [6] has introduced the notion of $b I$-open sets and $b I$-continuous functions in topological spaces. After

Received: May 30, 2015

(c) 2015 Academic Publications, Ltd. url: www.acadpubl.eu 
that Akdag [3], Ekici [7] and many others studied some more properties of these concepts and obtained several characterizations.

In this paper, we introduced $b I$-open sets and $b I$-continuous functions in ideal bitopological spaces and established several properties.

\section{Preliminaries}

Throughout this paper, $\left(X, \tau_{1}, \tau_{2}\right)$ denotes a bitopological space on which no separation axioms are assumed and $\left(X, \tau_{1}, \tau_{2}, I\right)$ be an ideal bitopological space. $i$-int $(A)$ and $j$-cl $(A)$ denotes the $i$-interior and $j$-closure of $A$ with respect to the topology $\tau_{i}$ and $\tau_{j}$ respectively, where $i, j \in\{1,2\}, i \neq j$.

Let $(X, \tau, I)$ be an ideal topological space. If $P(X)$ is the set of all subsets of $X$, then the operator $(.)^{*}: P(X) \longrightarrow P(X)$ is called the local function $([11])$ of $A$ with respect to the topology $\tau$ and ideal $I$ defined by $A^{*}(\tau, I)=\{x \in$ $X: B \cap A \notin I$, for every $B \in \tau(x)\}$, where $A \subset X$ and $\tau(x)=\{B \in \tau$ : $x \in B\}$. Simply we can write $A^{*}$ instead of $A^{*}(\tau, I)$ in case there is no chance for confusion. A Kuratowski closure operator for $\tau^{*}(I)$ which is finer than $\tau$ is defined by $c l^{*}(A)=A \cup A^{*}$. $i$-int $t^{*}(A)$ denotes the interior of $A$ in $\tau_{i}^{*}(I)$ and $i$-int $\left(A_{j}^{*}\right)$ denotes the interior of $A_{j}^{*}$ with respect to the topology $\tau_{i}$, where $A_{j}^{*}=\left\{x \in X: B \cap A \notin I\right.$, for every $\left.B \in \tau_{j}(x)\right\}$.

The following definitions are due to Al-Hawary and Al-Omari [4].

Definition 2.1. A subset $A$ of a bitopological space $\left(X, \tau_{1}, \tau_{2}\right)$ is said to be $(i, j)$-b-open if $A \subset i$-int $(j-c l(A)) \cup j$-cl $(i$-int $(A))$. The complement of an $(i, j)$-b-open set is $(i, j)$-b-closed.

Definition 2.2. A function $f:\left(X, \tau_{1}, \tau_{2}\right) \longrightarrow\left(X, \sigma_{1}, \sigma_{2}\right)$ is said to be $(i, j)$-b-continuous(respectively, $(i, j)$-b-irresolute) if $f^{-1}(V)$ is $(i, j)$-b-open in $X$, for every $\sigma_{i}$-open(respectively, $(i, j)$ - $b$-open) set $V$ of $Y$.

The following definition is due to Pervine [13].

Definition 2.3. A function $f:\left(X, \tau_{1}, \tau_{2}\right) \longrightarrow\left(X, \sigma_{1}, \sigma_{2}\right)$ is said to be pairwise continuous if the induced functions $f:\left(X, \tau_{1}\right) \longrightarrow\left(X, \sigma_{1}\right)$ and $f$ : $\left(X, \tau_{2}\right) \longrightarrow\left(X, \sigma_{2}\right)$ are both continuous.

\section{3. $(i, j)-b I-O p e n$ Sets}

Definition 3.1. A subset $A$ of an ideal bitopological space $\left(X, \tau_{1}, \tau_{2}, I\right)$ is said to be $(i, j)$-bI-open if $A \subset i$-int $\left(j-c l^{*}(A)\right) \cup j$-cl* $(i$-int $(A))$, where $i, j \in$ $\{1,2\}, i \neq j$. 
We denote the family of all $(i, j)-b I$-open sets of $\left(X, \tau_{1}, \tau_{2}, I\right)$ by $(i, j)$ $B I O(X)$. By $(i, j)$ we mean the pair of topologies $\left(\tau_{i}, \tau_{j}\right)$.

Remark 3.1. $B I O\left(X, \tau_{1}, \tau_{2}, I\right) \neq B I O\left(X, \tau_{1}\right) \cup B I O\left(X, \tau_{2}\right)$. It is clear from the following example.

Example 3.1. Let $X=\{p, q, r\}, \tau_{1}=\{\emptyset,\{p\}, X\}, \tau_{2}=\{\emptyset,\{q\}, X\}$ and $I=\{\emptyset,\{p\}\}$.

It can be easily shown that $\tau_{1}$-bI-open sets are $\{\emptyset,\{p\},\{p, q\},\{p, r\}, X\}$ and $\tau_{2}-b I$-open sets are $\{\emptyset,\{q\},\{p, q\},\{q, r\}, X\}$. But, $\left(\tau_{1}, \tau_{2}\right)$-bI-open sets are $\{\emptyset,\{p\},\{q\},\{p, q\},\{q, r\}, X\}$.

Remark 3.2. Every $(i, j)-b I$-open set is $(i, j)$-b-open. It can be easily proved by using the fact that $\tau^{*}(I)$ is finer than $\tau$.

But, the converse may not be true in general as shown by the following example.

Example 3.2. Let $X=\{p, q, r\}, \tau_{1}=\{\emptyset,\{q\}, X\}, \tau_{2}=\{\emptyset,\{p\},\{p, r\}, X\}$ and $I=\{\emptyset,\{p\}\}$. Now $\{p\}$ is $(1,2)$-b-open but not (1,2)-bI-open.

Remark 3.3. The intersection of two $(i, j)-b I$-open sets may not be a $(i, j)$-bI-open set is clear from the following example.

Example 3.3. Let $X=\{p, q, r\}, \tau_{1}=\{\emptyset,\{p, r\}, X\}, \tau_{2}=\{\emptyset,\{q, r\}, X\}$ and $I=\{\emptyset,\{p\}\}$. Then $\{p, q\}$ and $\{p, r\}$ is (1,2)-bI-open sets but $\{p, q\} \cap$ $\{p, r\}=\{p\}$ is not $(1,2)-b I$-open.

Theorem 3.1. Let $\left(X, \tau_{1}, \tau_{2}, I\right)$ be an ideal bitopological space and $A, B \subset$ $X$. If $A$ is $(i, j)$-bI-open set and $B \in \tau_{1} \cap \tau_{2}$, then $A \cap B$ is also $(i, j)$-bI-open.

Proof. Let $A$ is $(i, j)-b I$-open. Then $A \subset i$-int $\left(j-c l^{*}(A)\right) \cup j$-cl* $(i$-int $(A))$. Now

$$
\begin{aligned}
A & \cap B \subset\left\{i-\operatorname{int}\left(j-c l^{*}(A)\right) \cup j-c l^{*}(i-\operatorname{int}(A))\right\} \cap B \\
& =\left\{i-\operatorname{int}\left(j-c l^{*}(A)\right) \cap B\right\} \cup\left\{j-c l^{*}(i-\operatorname{int}(A)) \cap B\right\} \\
& =\left\{i-\operatorname{int}\left(A \cup A_{j}^{*}\right) \cap B\right\} \cup\left\{\left((i-\operatorname{int}(A)) \cup(i-\operatorname{int}(A))_{j}^{*}\right) \cap B\right\} \\
& \subset\left\{i-\operatorname{int}\left((A \cap B) \cup\left(A_{j}^{*} \cap B\right)\right)\right\} \cup\left\{((i-\operatorname{int}(A)) \cap B) \cup((i-\operatorname{int}(A)) \cap B)_{j}^{*}\right\} \\
& \subset\left\{i-\operatorname{int}\left((A \cap B) \cup(A \cap B)_{j}^{*}\right)\right\} \cup\left\{(i-\operatorname{int}(A \cap B)) \cup(i-\operatorname{int}(A \cap B))_{j}^{*}\right\} \\
& =i-\operatorname{int}\left(j-c l^{*}(A \cap B)\right) \cup j-c l^{*}(i-\operatorname{int}(A \cap B)) .
\end{aligned}
$$

Hence $A \cap B$ is $(i, j)$-bI-open. 
Theorem 3.2. Let $\left(X, \tau_{1}, \tau_{2}, I\right)$ be an ideal bitopological space. If $A_{\alpha} \in$ $(i, j)-B I O(X)$ for each $\alpha \in \wedge$, then $\bigcup\left\{A_{\alpha}: \alpha \in \wedge\right\} \in(i, j)-B I O(X)$ where $\wedge$ an index set.

Proof. Let $A_{\alpha} \in(i, j)-B I O(X)$. Then

$$
A_{\alpha} \subset i-\operatorname{int}\left(j-c l^{*}\left(A_{\alpha}\right)\right) \cup j-c l^{*}\left(i-\operatorname{int}\left(A_{\alpha}\right)\right)
$$

for each $\alpha \in \wedge$.

Thus

$$
\begin{aligned}
\bigcup_{\alpha \in \wedge} A_{\alpha} \subset & \bigcup_{\alpha \in \wedge}\left\{i-i n t\left(j-c l^{*}\left(A_{\alpha}\right)\right) \cup j-c l^{*}\left(i-i n t\left(A_{\alpha}\right)\right)\right\} \\
\subset & \bigcup_{\alpha \in \wedge}\left\{i-\operatorname{int}\left(A_{\alpha} \cup\left(A_{\alpha}\right)_{j}^{*}\right)\right) \cup\left\{\left(i-i n t\left(A_{\alpha}\right)\right) \cup\left(i-i n t\left(A_{\alpha}\right)\right)_{j}^{*}\right\} \\
\subset & \left\{i-\operatorname{int}\left(\left(\bigcup_{\alpha \in \wedge} A_{\alpha}\right) \cup\left(\bigcup_{\alpha \in \wedge}\left(A_{\alpha}\right)_{j}^{*}\right)\right)\right\} \\
& \cup\left\{\left(i-\operatorname{int}\left(\bigcup_{\alpha \in \wedge} A_{\alpha}\right)\right) \cup\left(\bigcup_{\alpha \in \wedge}\left(i-\operatorname{int}\left(A_{\alpha}\right)\right)_{j}^{*}\right\}\right. \\
\subset & \left\{i-\operatorname{int}\left(\left(\bigcup_{\alpha \in \wedge} A_{\alpha}\right) \cup\left(\bigcup_{\alpha \in \wedge} A_{\alpha}\right)_{j}^{*}\right)\right\} \\
& \cup\left\{i-i n t\left(\bigcup_{\alpha \in \wedge} A_{\alpha}\right) \cup\left(i-i n t\left(\bigcup_{\alpha \in \wedge} A_{\alpha}\right)\right)_{j}^{*}\right\} \\
\subset & i-\operatorname{int}\left(j-c l^{*}\left(\bigcup_{\alpha \in \wedge} A_{\alpha}\right)\right) \cup j-c l^{*}\left(i-i n t\left(\bigcup_{\alpha \in \wedge} A_{\alpha}\right)\right) .
\end{aligned}
$$

Hence $\bigcup_{\alpha \in \wedge} A_{\alpha}$ is $(i, j)$-bI-open.

Lemma 3.1. Let $\left(X, \tau_{1}, \tau_{2}, I\right)$ be an ideal bitopological space and $A, B$ are subsets of $X$ such that $B \subset A$. Then $B_{i}^{*}\left(\left.\tau_{i}\right|_{A},\left.I\right|_{A}\right)=B_{i}^{*}\left(\tau_{i}, I\right) \cap A$, for $i=1,2$.

Here we denote that for any subset $A$ of $\left(X, \tau_{1}, \tau_{2}, I\right),\left.\tau_{i}\right|_{A}$ is the relative topology on $A$ where $i=1,2$ and $\left.I\right|_{A}=\{A \cap I: I \in I\}$ is oviously an ideal on A.

Theorem 3.3. Let $\left(X, \tau_{1}, \tau_{2}, I\right)$ be an ideal bitopological space. If $A \in$ $(i, j)-B I O(X)$ and $B \in \tau_{1} \cap \tau_{2}$, then $A \cap B \in B I O\left(B,\left.\tau_{1}\right|_{B},\left.\tau_{2}\right|_{B},\left.I\right|_{B}\right)$.

Proof. Since $B \in \tau_{1} \cap \tau_{2}$, therefore $i$-int $t_{B}(P)=i$-int $(P)$, for any subset $P$ of $B$ and $i=1,2$. By using the fact and Lemma 3.1, we have

$A \cap B \subset\left\{i-\operatorname{int}\left(j-c l^{*}(A)\right) \cup j-c l^{*}(i-\operatorname{int}(A))\right\} \cap B$ 


$$
\begin{aligned}
= & \left.\left\{i-\operatorname{int}\left(j-c l^{*}(A)\right) \cap B\right\} \cup j-c l^{*}(i-i n t(A)) \cap B\right\} \\
= & \left.\left\{i-\operatorname{int}\left(A \cup A_{j}^{*}\right)\right) \cap B\right\} \cup\left\{\left((i-i n t(A)) \cup(i-\operatorname{int}(A))_{j}^{*}\right) \cap B\right\} \\
\subset & \left\{\left(\left(i-\operatorname{int}\left(A \cup A_{j}^{*}\right)\right) \cap B\right) \cap B\right\} \cup\{((i-i n t(A)) \cap B) \\
& \left.\left.\cup\left((i-\operatorname{int}(A))_{j}^{*} \cap B\right)\right) \cap B\right\} \\
\subset & \left\{\left(\left(i-\operatorname{int}\left(A \cup A_{j}^{*}\right)\right) \cap B\right) \cap B\right\} \cup\{((i-\operatorname{int}(A)) \cap B) \\
& \left.\left.\cup((i-\operatorname{int}(A)) \cap B)_{j}^{*}\right) \cap B\right\} \\
\subset & \left\{\left(\left(i-\operatorname{int}\left((A \cap B) \cup(A \cap B)_{j}^{*}\right)\right) \cap B\right)\right\} \cup\left\{\left(\left(i-\operatorname{int}_{B}(A \cap B)\right) \cap B\right)\right. \\
& \cup\left(\left(i-\operatorname{int} t_{B}(A \cap B)_{j}^{*}\right) \cap B\right\} \\
\subset & \left\{\left(\left(i-\operatorname{int}\left((A \cap B) \cup(A \cap B)_{j}^{*}\right)\right) \cap B\right)\right\} \cup\left\{\left(\left(i-i n t_{B}(A \cap B)\right)\right.\right. \\
& \left.\cup\left(i-\operatorname{int}_{B}(A \cap B)\right)_{j}^{*}\right)_{\left.\left(\tau_{1}\left|B, \tau_{2}\right| B, I \mid B\right)\right\}} \\
= & \left\{i-i n t_{B}\left(j-c l_{B}^{*}(A \cap B)\right)\right\} \cup\left\{j-c l_{B}^{*}\left(i-i n t_{B}(A \cap B)\right)\right\} .
\end{aligned}
$$

Hence $A \cap B \in B I O\left(B,\left.\tau_{1}\right|_{B},\left.\tau_{2}\right|_{B},\left.I\right|_{B}\right)$.

Definition 3.2. A subset $A$ of an ideal bitopological space $\left(X, \tau_{1}, \tau_{2}, I\right)$ is said to be $(i, j)$-bI-closed if its complement is $(i, j)$-bI-open.

Theorem 3.4. Let $\left(X, \tau_{1}, \tau_{2}, I\right)$ be an ideal bitopological space. Then a subset $A$ of $\left(X, \tau_{1}, \tau_{2}, I\right)$ is $(i, j)$-bI-closed if $i$-cl $\left(j\right.$-int $\left.t^{*}(A)\right) \cap j$-int $t^{*}(i$-cl $(A)) \subset A$, where $i, j \in\{1,2\}, i \neq j$.

Proof. It is clear from the definition.

Theorem 3.5. Let $\left(X, \tau_{1}, \tau_{2}, I\right)$ be an ideal bitopological space. If a subset $A$ of $X$ is $(i, j)$-bI-closed, then $i$-cl* $(j$-int $(A)) \cap j$-int $\left(i\right.$-cl $\left.l^{*}(A)\right) \subset A$, where $i, j \in\{1,2\}, i \neq j$.

Proof. Let $A$ is $(i, j)$-bI-closed. Then $X \backslash A$ is $(i, j)$-bI-open. Since $\tau_{i}^{*}$ is finer than $\tau_{i}(i=1,2)$, we have

$$
\begin{aligned}
X \backslash A & \subset i-\operatorname{int}\left(j-c l^{*}(X \backslash A)\right) \cup j-c l^{*}(i-\operatorname{int}(X \backslash A)) \\
& \subset i-\operatorname{int}(j-c l(X \backslash A)) \cup j-c l(i-\operatorname{int}(X \backslash A)) \\
& =\{X \backslash(i-c l(j-\operatorname{int}(A)))\} \cup\{X \backslash(j-\operatorname{int}(i-c l(A)))\} \\
& \subset\left\{X \backslash\left(i-c l^{*}(j-\operatorname{int}(A))\right)\right\} \cup\left\{X \backslash\left(j-\operatorname{int}\left(i-c l^{*}(A)\right)\right)\right\} \\
& =X \backslash\left\{\left(i-c l^{*}(j-\operatorname{int}(A))\right) \cap\left(j-\operatorname{int}\left(i-c l^{*}(A)\right)\right)\right\} .
\end{aligned}
$$

Hence $i$-cl $l^{*}(j$-int $(A)) \cap j$-int $\left(i-c l^{*}(A)\right) \subset A$.

Theorem 3.6. Let $\left(X, \tau_{1}, \tau_{2}, I\right)$ be an ideal bitopological space and $A \subset X$. Then: 
(a) If $I=\emptyset$, then $A$ is $(i, j)$-bI-open if and only if $A$ is $(i, j)$-b-open.

(b) If $I=P(X)$, then $A$ is $(i, j)$-bI-open if and only if $A \in \tau_{1} \cap \tau_{2}$.

Proof. (a) It follows from the fact that if $I=\emptyset$, then $A^{*}=\operatorname{cl}(A)$, for every subset $A$ of $X$.

(b) It follows from the fact that for every subset $A$ of $X$, if $I=P(X)$ then $A^{*}=\emptyset$.

Definition 3.3. Let $\left(X, \tau_{1}, \tau_{2}, I\right)$ be an ideal bitopological space and $A \subset$ $X$. Then

(a) The $(i, j)-b I$-interior of $A$, is defined as the union of all $(i, j)$-bI-open sets contained in $A$ and is denoted by $(i, j)-b I-i n t(A)$.

(b) The $(i, j)$-bI-closure of $A$, is defined as the intersection of all $(i, j)-b I$ closed sets containing $A$ and is denoted by $(i, j)-b I-c l(A)$.

Theorem 3.7. Let $A$ be a subset of an ideal bitopological space $\left(X, \tau_{1}, \tau_{2}, I\right)$. Then:

(a) $(i, j)-b I-\operatorname{int}(A)$ is $(i, j)$-bI-open.

(b) $(i, j)-b I-c l(A)$ is $(i, j)$-bI-closed.

(c) $A$ is $(i, j)$-bI-open if and only if $A=(i, j)-b I$-int $(A)$.

(d) $A$ is $(i, j)-b I$-closed if and only if $A=(i, j)-b I-c l(A)$.

Proof. The proof of $(a)-(d)$ are obvious.

Theorem 3.8. Let $\left(X, \tau_{1}, \tau_{2}, I\right)$ be an ideal bitopological space and $A \subset X$. Then a point $x \in(i, j)-b I-c l(A)$ if and only if $A \cap B \neq \emptyset$, for every $(i, j)$-bI-open set $B$ of $X$ containing $x$.

Proof. Suppose that $x \in(i, j)-b I-c l(A)$ and $B$ be a $(i, j)-b I$-open set containing $x$.Assume that $A \cap B=\emptyset$.Then $A \subset X \backslash B$, where $X \backslash B$ is $(i, j)$-bI-closed set. This implies that $x \in(i, j)-b I-c l(A) \subset(i, j)-b I-c l(X \backslash B)=X \backslash B$. Thus $x \in X \backslash B$, which is a contradiction. Hence $A \cap B \neq \emptyset$.

Conversely suppose that $A \cap B \neq \emptyset$, for every $(i, j)$-bI-open set $B$ of $X$ containing $x$.Let $x \notin(i, j)$-bI-cl $(A)$. Then there exists a $(i, j)$-bI-closed set $G$ of $X$ such that $A \subset G$ and $x \notin G$. Therefore $x \in X \backslash G$, where $X \backslash G$ is $(i, j)-b I$ open and $(X \backslash G) \cap A=\emptyset$. Which is a contradiction to the assumption. Hence $x \in(i, j)-b I-c l(A)$.

Theorem 3.9. Let $A$ be a subset of an ideal bitopological space $\left(X, \tau_{1}, \tau_{2}, I\right)$. Then 
(a) $(i, j)-b I-c l(X \backslash A)=X \backslash(i, j)-b I-i n t(A)$.

(b) $(i, j)-b I-i n t(X \backslash A)=X \backslash(i, j)-b I-c l(A)$.

Proof. (a) Let $x \notin(i, j)-b I$-cl $(X \backslash A)$. Then there exists an $(i, j)$-bI-open set $B$ of $X$ containing $x$ such that $B \cap(X \backslash A)=\emptyset$. We have $x \in B$, therefore $x \notin$ $X \backslash A$ and so $x \in A$. Thus $x \in B \subset A$ and so $x \in(i, j)$-bI-int $(A)$. Which implies that $x \notin X \backslash(i, j)$-bI-int $(A)$. Hence $X \backslash(i, j)$-bI-int $(A) \subset(i, j)$-bI-cl $(X \backslash A)$. Next let $x \notin X \backslash(i, j)$-bI-int $(A)$. Then $x \in(i, j)$-bI-int $(A)$ and so there exists an $(i, j)$-bI-open set $B$ of $X$ such that $x \in B \subset A$. Thus $B \cap(X \backslash A)=\emptyset$ and $x \notin(i, j)$-bI-cl $(X \backslash A)$. Therefore $(i, j)$-bI-cl $(X \backslash A) \subset X \backslash(i, j)$-bI-int $(A)$. Hence the result follows.

(b) Similar to the proof of (a).

Definition 3.4. Let $\left(X, \tau_{1}, \tau_{2}, I\right)$ be an ideal bitopological space. Then a subset $A$ of $X$ is said to be an $(i, j)$-bI-neighbourhood of a point $x$ of $X$ if there exists a $(i, j)-b I$-open set $B$ such that $x \in B \subset A$.

Theorem 3.10. Let $\left(X, \tau_{1}, \tau_{2}, I\right)$ be an ideal bitopological space and $A \subset$ $X$. Then $A$ is $(i, j)-b I$-open if and only if it is an $(i, j)$-bI-neighbourhood of each of its points.

Proof. Let $A$ be an $(i, j)-b I$-open set of $\left(X, \tau_{1}, \tau_{2}, I\right)$. So by definition, we have $A$ is an $(i, j)-b I$-neighbourhood of each of its points, since for every $x \in A$, we have $x \in A \subset A$ and $A$ is $(i, j)$-bI-open.Conversely assume that $A$ is an $(i, j)-b I$-neighbourhood of each of its points. Then for every $x \in A$, there exists an $(i, j)-b I$-open set $B_{x}$ of $\left(X, \tau_{1}, \tau_{2}, I\right)$ such that $x \in B_{x} \subset A$. Then $A=\bigcup\left\{B_{x}: x \in A\right\}$. Since arbitrary union of $(i, j)$-bI-open sets is $(i, j)$-bIopen, therefore we have $A$ is $(i, j)$-bI-open in $X$.

\section{4. $(i, j)$-bI-Continuous Functions}

Definition 4.1. A function $f:\left(X, \tau_{1}, \tau_{2}, I\right) \longrightarrow\left(Y, \sigma_{1}, \sigma_{2}\right)$ is said to be $(i, j)$-bI-continuous if the inverse image of every $\sigma_{i}$-open set in $Y$ is $(i, j)$-bI-open in $X$, where $i, j=1,2$ and $i \neq j$.

Remark 4.1. Every $(i, j)$-bI-continuous function is $(i, j)$-b-continuous. But the converse may not be true in general as shown in the following example.

Example 4.1. Let $X=\{p, q, r\}, \tau_{1}=\{\emptyset,\{q\}, X\}, \tau_{2}=\{\emptyset,\{p\},\{q, r\}, X\}$ and $I=\{\emptyset,\{p\}\}$. Then the identity function $f:\left(X, \tau_{1}, \tau_{2}, I\right) \longrightarrow\left(X, \sigma_{1}, \sigma_{2}\right)$ is $(1,2)$-b-continuous but not $(1,2)-b I$-continuous. 
Theorem 4.1. The following statements are equivalent for the function $f:\left(X, \tau_{1}, \tau_{2}, I\right) \longrightarrow\left(Y, \sigma_{1}, \sigma_{2}\right)$.

(a) $f$ is $(i, j)$-bI-continuous.

(b) For all $x \in X$ and every $\sigma_{i}$-open set $B$ of $Y$ containing $f(x)$, there exists a $(i, j)$-bI-open set $A$ of $X$ containing $x$ such that $f(A) \subset B$.

(c) Inverse image of every $\sigma_{i}$-closed set of $Y$ is $(i, j)$-bI-closed set in $X$.

(d) $f((i, j)-b I-c l(A)) \subset \sigma_{i}-c l(f(A))$, for every subset $A$ of $X$.

(e) $(i, j)-b I-c l\left(f^{-1}(B)\right) \subset f^{-1}\left(\sigma_{i}-c l(B)\right)$, for every subset $B$ of $Y$.

$(f) f^{-1}\left(\sigma_{i}-i n t(G)\right) \subset(i, j)-b I-i n t\left(f^{-1}(G)\right)$, for every subset $G$ of $Y$.

Proof. $(a) \Rightarrow(b)$ Let $B$ be a $\sigma_{i}$-open set in $Y$ such that $f(x) \in B$. Since $f$ is $(i, j)$-bI-continuous, therefore $f^{-1}(B)$ is $(i, j)$-bI-open in $X$. Let $A=f^{-1}(B)$. Then $f(x) \in f(A) \subset B$.

$(b) \Rightarrow(a)$ Let $B$ be a $\sigma_{i}$-open set in $Y$ and $x \in f^{-1}(B)$.Then we have $f(x) \in B$. By (b), there exists an $(i, j)$-bI-open set $A_{x}$ in $X$ containing $x$ such that $f\left(A_{x}\right) \subset B$. Therefore $x \in A_{x} \in f^{-1}(B)$. Consequently, $f^{-1}(B)$ is $(i, j)$-bI-open in $X$. Hence $f^{-1}(B)$ is $(i, j)$-bI-continuous.

$(a) \Rightarrow(c)$ It is obvious.

$(c) \Rightarrow(d)$ Let $A \subset X$. Since $\sigma_{i}-c l(f(A))$ is $\sigma_{i}$-closed set in $Y$, therefore by (c) we have $f^{-1}\left(\sigma_{i}-c l(f(A))\right.$ is $(i, j)$-bI-closed set in $X$. Also, $A \subset f^{-1}\left(\sigma_{i}-c l(f(A))\right.$ and $(i, j)-b I-c l(A)$ is the smallest $(i, j)-b I$-closed set containing $A$. Therefore $(i, j)-b I-c l(A) \subset f^{-1}\left(\sigma_{i^{-}} c l(f(A))\right.$. This implies that $f((i, j)-b I-c l(A)) \subset \sigma_{i^{-}}$ $c l(f(A))$.

$(d) \Rightarrow(e)$ Let $B \subset Y$. Then $f^{-1}(B)$ is a subset of $X$. By $(\mathrm{d}), f((i, j)$ $\left.b I-c l\left(f^{-1}(B)\right)\right) \subset \sigma_{i}-c l\left(f\left(f^{-1}(B)\right)\right) \subset \sigma_{i}-c l(B)$. Hence $(i, j)-b I-c l\left(f^{-1}(B)\right) \subset$ $f^{-1}\left(\sigma_{i}-c l(B)\right)$.

$(e) \Rightarrow(c)$ Let $B$ be a $\sigma_{i}$-closed set in $Y$. By $(\mathrm{e}),(i, j)-b I-c l\left(f^{-1}(B)\right) \subset$ $f^{-1}\left(\sigma_{i}-c l(B)\right)=f^{-1}(B)$. Therefore $f^{-1}(B)=(i, j)-b I-c l\left(f^{-1}(B)\right)$ and so $f^{-1}(B)$ is $(i, j)-b I$-closed in $X$.

$(a) \Rightarrow(f)$ Let $G$ be a $\sigma_{i^{-}}$open subset of $Y$. By $(\mathrm{a}), f^{-1}(G)=f^{-1}\left(\sigma_{i^{-}}\right.$ $\operatorname{int}(G))$ is $(i, j)$-bI-open in $X$. Then $f^{-1}\left(\sigma_{i}-i n t(G)\right) \subset(i, j)-b I-i n t\left(f^{-1}\left(\sigma_{i^{-}}\right.\right.$ $\operatorname{int}(G))) \subset(i, j)-b I-i n t\left(f^{-1}(G)\right)$.

$(f) \Rightarrow(a)$ Let $B$ be a $\sigma_{i^{-}}$open subset of $Y$. Then $f^{-1}(B)=f^{-1}\left(\sigma_{i^{-}}\right.$ $\operatorname{int}(B)) \subset(i, j)$-bI-int $\left(f^{-1}(B)\right)$. Therefore $f^{-1}(B)$ is $(i, j)$-bI-open in $X$ and so $f$ is $(i, j)-b I$-continuous. 
Theorem 4.2. Let

$$
f:\left(X, \tau_{1}, \tau_{2}, I\right) \longrightarrow\left(Y, \sigma_{1}, \sigma_{2}, J\right) \text { and } g:\left(Y, \sigma_{1}, \sigma_{2}, J\right) \longrightarrow\left(Z, \theta_{1}, \theta_{2}\right)
$$

be two functions, where $I$ and $J$ are two ideals in $X$ and $Y$ respectively. If $f$ is $(i, j)$-bI-continuous and $g$ is pairwise continuous, then $g_{0} f$ is $(i, j)-b I$ continuous.

Proof. Let $C$ be a $\theta_{i}$-open set in $Z$. Since $g$ is pairwise continuous, therefore $g^{-1}(C)$ is $\sigma_{i}$-open set in $Y$. Also, $f$ is $(i, j)$-bI-continuous, so $\left(g_{0} f\right)^{-1}(C)=$ $f^{-1}\left(g^{-1}(C)\right)$ is $(i, j)$-bI-open in $X$. Hence $g_{0} f$ is $(i, j)-b I$-continuous.

Theorem 4.3. Let $f:\left(X, \tau_{1}, \tau_{2}, I\right) \rightarrow\left(Y, \sigma_{1}, \sigma_{2}\right)$ is $(i, j)$-bI-continuous and $A \subset X$. If $A \in \tau_{1} \cap \tau_{2}$, then the restriction function $\left.f\right|_{A}:\left(A,\left.\tau_{1}\right|_{A},\left.\tau_{2}\right|_{A},\left.I\right|_{A}\right) \rightarrow$ $\left(Y, \sigma_{1}, \sigma_{2}\right)$ is $(i, j)$-bI-continuous.

Proof. Let $B$ be a $\sigma_{i}$-open subset of $Y$. Since $f$ is $(i, j)$-bI-continuous, therefore $f^{-1}(B)$ is $(i, j)$-bI-open set in $X$. Also, $A \in \tau_{1} \cap \tau_{2}$, therefore by theorem 3.3, we have $A \cap f^{-1}(B) \in B I O\left(A,\left.\tau_{1}\right|_{A},\left.\tau_{2}\right|_{A},\left.I\right|_{A}\right)$. Again

$$
\left(\left.f\right|_{A}\right)^{-1}(B)=A \cap f^{-1}(B)
$$

and so $\left(\left.f\right|_{A}\right)^{-1}(B) \in B I O\left(A,\left.\tau_{1}\right|_{A},\left.\tau_{2}\right|_{A},\left.I\right|_{A}\right)$. Hence $\left.f\right|_{A}:\left(A,\left.\tau_{1}\right|_{A},\left.\tau_{2}\right|_{A},\left.I\right|_{A}\right)$ $\rightarrow\left(Y, \sigma_{1}, \sigma_{2}\right)$ is $(i, j)$-bI-continuous.

Theorem 4.4. Let $f:\left(X, \tau_{1}, \tau_{2}, I\right) \longrightarrow\left(Y, \sigma_{1}, \sigma_{2}\right)$ be a function. If a function $g:\left(X, \tau_{1}, \tau_{2}, I\right) \longrightarrow\left(X \times Y, \sigma_{1} \times \sigma_{2}\right)$, defined by $g(x)=(x, f(x))$ for each $x \in X$ is $(i, j)$-bI-continuous, then $f$ is $(i, j)$-bI-continuous.

Proof. Suppose that $g$ is $(i, j)$-bI-continuous. Let $x \in X$ and $B$ be a $\sigma_{i}$-open subset of $Y$ containing $f(x)$. Then $X \times B$ is $\tau_{i} \times \sigma_{i}$-open in $X \times Y$. By the $(i, j)$-bI-continuity of $g$, there exists an $(i, j)$-bI-open set $A$ in $X$ containing $x$ such that $f(A) \subset X \times Y$, by theorem 4.1. So $f(A) \subset B$. Hence $f$ is $(i, j)-b I$ continuous.

Definition 4.2. (see [13]) A bitopological space $\left(X, \tau_{1}, \tau_{2}\right)$ is said to be pairwise connected if it cannot be expressed as the union of two nonempty disjoint sets $A$ and $B$ such that $A$ is $\tau_{i}$-open and $B$ is $\tau_{j}$-open, where $i, j=\{1,2\}$.

Definition 4.3. An ideal bitopological space $\left(X, \tau_{1}, \tau_{2}, I\right)$ is said to be $(i, j)$ $b I$-connected if there exists a nonempty $(i, j)$-bI-open set $A$ and a nonempty $(j, i)-b I$-open set $B$ in $X$ such that $X \neq A \cup B$ and $A \cap B=\emptyset$.

Theorem 4.5. Let $f:\left(X, \tau_{1}, \tau_{2}, I\right) \longrightarrow\left(Y, \sigma_{1}, \sigma_{2}\right)$ be a function. If $f$ is $(i, j)$-bI-continuous surjection and $X$ is $(i, j)$-bI-connect-ed, then $Y$ is pairwise connected. 
Proof. Assume that $Y$ is not pairwise connected. Then there exists a nonempty $\sigma_{i}$-open set $A$ and a nonempty $\sigma_{j}$-open set $B$ such that $Y=A \cup B$ and $A \cap B=\emptyset$. Since $f$ is $(i, j)$-bI-continuous, therefore $f^{-1}(A)$ is $(i, j)-b I$ open in $X$ and $f^{-1}(B)$ is $(j, i)$-bI-open in $X$. Since $A \cap B=\emptyset$ and $f$ is surjective, therefore $f^{-1}(A) \cap f^{-1}(B)=\emptyset$. Thus $f^{-1}(Y)=f^{-1}(A) \cup f^{-1}(B)$ and $f^{-1}(A) \neq \emptyset$ and $f^{-1}(B) \neq \emptyset$. This implies that $X=f^{-1}(A) \cup f^{-1}(B)$. Hence $X$ is not $(i, j)$-bI-connected, which is a contradiction.

Lemma 4.1([12]). For any function $f:(X, \tau, I) \longrightarrow(Y, \sigma), f(I)$ is an ideal on $Y$.

Definition 4.4. An ideal bitopological space $\left(X, \tau_{1}, \tau_{2}, I\right)$ is said to be $(i, j)$ - $I$-compact if for every open cover $\left\{C_{\alpha}: \alpha \in \wedge\right\}$ by $\tau_{i}$-open sets of $X$, there exists a finite subset $\wedge_{0}$ of $\wedge$ such that $X \backslash \bigcup\left\{C_{\alpha}: \alpha \in \wedge\right\} \in I$, where $i, j=\{1,2\}$ and $i \neq j$.

Definition 4.5. An ideal bitopological space $\left(X, \tau_{1}, \tau_{2}, I\right)$ is said to be $(i, j)$-bI-compact if for every open cover $\left\{C_{\alpha}: \alpha \in \wedge\right\}$ by $(i, j)$-bI-open sets of $X$, there exists a finite subset $\wedge_{0}$ of $\wedge$ such that $X \backslash \bigcup\left\{C_{\alpha}: \alpha \in \wedge\right\} \in I$, where $i, j=\{1,2\}$ and $i \neq j$.

Theorem 4.6. Let $f:\left(X, \tau_{1}, \tau_{2}, I\right) \longrightarrow\left(Y, \sigma_{1}, \sigma_{2}\right)$ be a function. If $f$ : $\left(X, \tau_{1}, \tau_{2}, I\right) \longrightarrow\left(Y, \sigma_{1}, \sigma_{2}\right)$ is $(i, j)$-bI-continuous surjection and $X$ is $(i, j)-b I$ compact, then $\left(Y, \sigma_{1}, \sigma_{2}, f(I)\right)$ is $(i, j)$ - $f(I)$-compact.

Proof. Let $\left\{C_{\alpha}: \alpha \in \wedge\right\}$ be an open cover of $Y$ by $\sigma_{i}$-open sets in $Y$. Since $f$ is $(i, j)$-bI-continuous, therefore $\left\{f^{-1}\left(C_{\alpha}\right): \alpha \in \wedge\right\}$ be an open cover of $X$ by $(i, j)$-bI-open sets in $X$. Also, $\left(X, \tau_{1}, \tau_{2}, I\right)$ is $(i, j)$-bI-compact. Therefore there exists a finite subset $\wedge_{0}$ of $\wedge$ such that $X \backslash \bigcup\left\{f^{-1}\left(C_{\alpha}\right): \alpha \in \wedge_{0}\right\} \in I$. Thus $Y \backslash \bigcup\left\{C_{\alpha}: \alpha \in \wedge_{0}\right\}=f\left(X \backslash \bigcup\left\{f^{-1}\left(C_{\alpha}\right): \alpha \in \wedge_{0}\right\} \in f(I)\right.$. Hence $\left(Y, \sigma_{1}, \sigma_{2}, f(I)\right)$ is $(i, j)-f(I)$-compact.

Definition 4.6. A function $f:\left(X, \tau_{1}, \tau_{2}, I\right) \longrightarrow\left(Y, \sigma_{1}, \sigma_{2}\right)$ is said to be $(i, j)$ - $b I$-irresolute if the inverse image of every $(i, j)$-b-open set in $Y$ is $(i, j)$-bIopen in $X$, where $i, j=1,2$ and $i \neq j$.

Theorem 4.7. The following statements are equivalent for the function $f:\left(X, \tau_{1}, \tau_{2}, I\right) \longrightarrow\left(Y, \sigma_{1}, \sigma_{2}\right)$.

(a) $f$ is $(i, j)$-bI-irresolute.

(b) For all $x \in X$ and every $(i, j)$-b-open set $B$ of $Y$ containing $f(x)$, there exists an $(i, j)$-bI-open set $A$ of $X$ containing $x$ such that $f(A) \subset B$.

(c) Inverse image of every $(i, j)$-b-closed set of $Y$ is $(i, j)$-bI-closed set in $X$. 
Theorem 4.8. Let

$$
f:\left(X, \tau_{1}, \tau_{2}, I\right) \longrightarrow\left(Y, \sigma_{1}, \sigma_{2}, J\right) \text { and } g:\left(Y, \sigma_{1}, \sigma_{2}, J\right) \longrightarrow\left(Z, \theta_{1}, \theta_{2}\right)
$$

be two functions, where $I$ and $J$ are two ideals in $X$ and $Y$ respectively.

(a) If $f$ is $(i, j)$-bI-irresolute and $g$ is $\left(i, j\right.$-b-irresolute, then $g_{0} f$ is $(i, j)-b I$ irresolute.

(b) If $f$ is $(i, j)$-bI-irresolute and $g$ is $\left(i, j\right.$-b-continuous, then $g_{0} f$ is $(i, j)-b I$ continuous.

Proof. (a) Let $C$ be an $(i, j)$-b-open set in $Z$. Since $g$ is $(i, j$-b-irresolute, therefore $g^{-1}(C)$ is $(i, j)$-b-open set in $Y$. Also, $f$ is $(i, j)$-bI-irresolute, so $\left(g_{0} f\right)^{-1}(C)=f^{-1}\left(g^{-1}(C)\right)$ is $(i, j)$-bI-open in $X$. Hence $g_{0} f$ is $(i, j)$-bI-irresolute.

(b) Let $C$ be a $\theta_{i}$-open set in $Z$. Since $g$ is $(i, j$-b-continuous, therefore $g^{-1}(C)$ is $(i, j)$-b-open set in $Y$. Also, $f$ is $(i, j)$-bI-irresolute, so $\left(g_{0} f\right)^{-1}(C)=$ $f^{-1}\left(g^{-1}(C)\right)$ is $(i, j)$-bI-open in $X$. Hence $g_{0} f$ is $(i, j)$ - $b I$-continuous.

Theorem 4.9. Let $f:\left(X, \tau_{1}, \tau_{2}, I\right) \longrightarrow\left(Y, \sigma_{1}, \sigma_{2}\right)$ is $(i, j)$-bI-irresolute and $A \subset X$. If $A \in \tau_{1} \cap \tau_{2}$, then the restriction function $\left.f\right|_{A}:\left(A,\left.\tau_{1}\right|_{A},\left.\tau_{2}\right|_{A},\left.I\right|_{A}\right) \longrightarrow$ $\left(Y, \sigma_{1}, \sigma_{2}\right)$ is $(i, j)$-bI-irresolute.

Proof. Let $B$ be an $(i, j)$-b-open subset of $Y$. Since $f$ is $(i, j)$-bI-irresolute, therefore $f^{-1}(B)$ is $(i, j)-b I$-open set in $X$. Also, $A \in \tau_{1} \cap \tau_{2}$, therefore by theorem 3.3, we have $A \cap f^{-1}(B) \in B I O\left(A,\left.\tau_{1}\right|_{A},\left.\tau_{2}\right|_{A},\left.I\right|_{A}\right)$. Again

$$
\left(\left.f\right|_{A}\right)^{-1}(B)=A \cap f^{-1}(B)
$$

and so

$$
\left(\left.f\right|_{A}\right)^{-1}(B) \in B I O\left(A,\left.\tau_{1}\right|_{A},\left.\tau_{2}\right|_{A},\left.I\right|_{A}\right) .
$$

Hence

$$
\left.f\right|_{A}:\left(A,\left.\tau_{1}\right|_{A},\left.\tau_{2}\right|_{A},\left.I\right|_{A}\right) \rightarrow\left(Y, \sigma_{1}, \sigma_{2}\right)
$$

is $(i, j)$-bI-irresolute.

\section{References}

[1] M. E. Abd El-Monsef, E. F. Lashien and A. A. Nasef, On I-open sets and I-continuous functions, Kyungpook Math. J., 32, No.1 (1992), 21-30.

[2] A. Acikgoz, S. Yuksel and T. Noiri, $\alpha-I$-Preirresolute Functions and $\beta-I$ Preirresolute Functions, Bull. Malays. Math. Sci. Soc., 28, No. 1-2 (2005), $1-8$. 
[3] M. Akdag, On $b$ - $I$-open sets and $b$ - $I$-continuous functions, Int. J. Math. Math. Sci., 22 (2007), 27-32, doi: 1155/2007/75721.

[4] T. Al-Hawary and A. Al-Omari, $b$-open and b-continuity in Bitopological Spaces, Al-Manarah, 13, No. 3 (2007), 89-101.

[5] D. Andrijevic, On b-open sets, Mat. Vesnik, 48 (1996), 59 - 64.

[6] A. Caksu Guler and G. Aslim, b-I-open sets and decomposition of continuity via idealization, Proc. Inst. Math., National Academy of Sciences of Azerbaijan, 22 (2005), 27-32.

[7] E. Ekici, On Pre- $I$-open sets, Semi- $I$-open sets and $b$ - $I$-open sets in Ideal Topological Spaces, Acta Universitasis Apulensis, 30 (2012), 293-303.

[8] E. Hatir and T. Noiri, On $\beta$ - $I$-open sets and a decomposition of almost I-continuity, Bull. Malays. Math. Sci. Soc., 29, No. 1-2 (2006), 119-124.

[9] D. Jankovic and T.R. Hamlett, New topologies from old via ideals, Amer. Math. Monthly, 97 (1990), 295-310, doi: 10.2307/232 4512.

[10] J.C. Kelly, Bitopological spaces, Proc. London Math. Soc., 13, No. 3 (1963), 71-89.

[11] K. Kuratowski, Topology, Academic Press, New York,(1966).

[12] R. L. Newcomb, Topologies which are compact modulo an ideal, Ph. D dissertation, University of California, Santa Barbara, Calif, USA, 1967.

[13] W. J. Pervine, Connectedness in bitopological spaces, Indag. Math., 29 (1967), 369-372.

[14] M.S. Sarsak and N. Rajesh, Special Functions on Bitopological Spaces, Internat. Math. Forum, 36, No. 4 (2009), 1775-1782.

[15] R. Vaidyanathaswamy, The localisation theory in set topology, Proc. Indian. Acad. Sci., 20 (1945), 51-61. 